\title{
Transfer C-H Borylation of Alkenes under Rh(I)-Catalysis: Insight into the Mechanism, Selectivity-Control \& Synthetic Capacity
}

\author{
Lukas Veth, ${ }^{\star}$ Hanusch A. Grab, ${ }^{\star}$ Sebastián Martínez, ${ }^{\star}$ Cyril Antheaume, and Paweł Dydio* \\ University of Strasbourg, CNRS, ISIS UMR 7006, 8 allée Gaspard Monge, 67000 Strasbourg, France
}

\begin{abstract}
Transfer C-H borylation of alkenes bears the potential to unlock a range of attractive transformations for modular synthesis and late-stage derivatization of complex molecules. However, its scarce precedence associated with a limited mechanistic understanding hinders the development of practical synthetic protocols. Here we report a $\mathrm{Rh}(\mathrm{I})$-catalyzed transfer $\mathrm{C}-\mathrm{H}$ borylation reaction that is founded on an unprecedented yet crucial elementary step of the $\beta$-boryl elimination engaging the $\mathrm{Rh}(\mathrm{I})$-( $\beta$-borylalkyl) intermediate. A thorough mechanistic investigation involving a series of catalytic
\end{abstract}

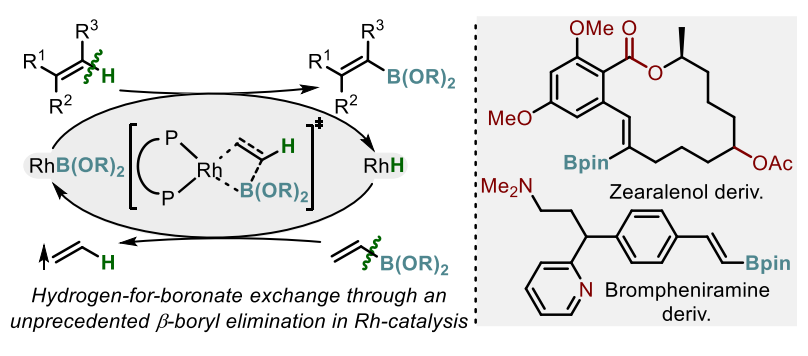
and stoichiometric experiments and complementary computational studies revealed that this step proceeds with a considerably low free energy barrier, further elucidated the full catalytic cycle, and provided insight into the features controlling the activity and the selectivity. Driven by this mechanistic understanding, we devised a protocol that is compatible with a plethora of functional groups, including often problematic motifs, and applicable not only to terminal but also internal alkenes and varied electronic and steric properties. The method proved also to be effective in complex settings of the late-stage borylation of derivatives of macrocyclic mycoestrogen Zearalenol, bioactive Brompheniramine, Chlorpromazine, and CD3254, and the synthesis of the boronic acid bioisostere of the drug Ozagrel. Besides the valuable new method, these mechanistic investigations set the stage for the development of other hydrogen-for-functional group exchange reactions undergoing a similar pathway.

With the multitude of methods to convert a $\mathrm{C}-\mathrm{B}$ bond into a $\mathrm{C}-\mathrm{C}$ or $\mathrm{C}-\mathrm{X}$ bond, ${ }^{1-4}$ the ability to install a boronic ester group on available starting materials is fundamental for both modular synthesis and late-stage functionalization of complex molecules. $^{5}$ Considering the presence of alkene motifs in various synthetic and natural compounds of high structural complexity, methods for their selective borylation are particularly appealing (Figure 1a). ${ }^{6-8}$ In that context, due to its mild conditions, borylation through alkene cross-metathesis ${ }^{9-13}$ has found numerous applications in fine-chemical synthesis (Figure 1b). ${ }^{14-18}$ However, when the initial carbon skeleton is to be maintained, the approach is unsuitable for the borylation of substrates bearing internal alkenes, e.g., many (macrocyclic) natural products. ${ }^{19-36}$ Besides, congested double bonds of low reactivity ${ }^{37}$ as well as starting materials bearing amines, ${ }^{38,39}$ heterocycles, ${ }^{40,41}$ alkynes, ${ }^{42}$ or electrophilic motifs, such as aldehydes and Michael acceptors, ${ }^{43,44}$ are often problematic, due to either catalyst inhibition by strong coordination, catalyst decomposition, or impeding side-reactions.

With the overarching goal of formulating an approach that bears the potential to address current synthetic limitations, we surmised that functional group transfer catalysis ${ }^{45}$ could enrich the scope of strategies for alkene borylation (Figure 1c). Ideally, it would be applicable to both terminal and internal alkene starting materials, while at the same time employing a benign reagent as a boryl group donor, ensuring a tolerance toward a broad range of functional groups. ${ }^{46}$ Remarkably, Marciniec ${ }^{47,48}$ and $\mathrm{Wu}^{36}$ demonstrated a boryl group transfer reactivity between boryl group donors and simple alkenes in the presence of $\left[\mathrm{RuHCl}(\mathrm{CO})\left(\mathrm{PCy}_{3}\right)_{2}\right]$ or $\left[\mathrm{Cp}_{2} \mathrm{ZrH}_{2}\right]$. However, the high reactivity of the catalytic $\mathrm{Ru}(\mathrm{II})$ - and $\mathrm{Zr}$ (II)-hydride species toward common functional groups, including alkynes, aldehydes, or ketones, impedes their applicability in fine-chemical synthesis. Overall, with scarce precedence and limited mechanistic insight, ${ }^{49}$ both the full potential and rational development of synthetic methods of boryl group transfer remain elusive.

We hypothesized that phosphine-Rh(I)-complexes would be the catalysts of choice for a chemoselective boryl group transfer that would occur in a catalytic cycle involving a series of alkene insertion and $\beta$-elimination steps, as shown in Figure 1c. Our reasoning was as follows: (i) In stoichiometric experiments, a $\mathrm{Rh}(\mathrm{I})$-boryl complex reacted with styrene to form $\mathrm{Rh}(\mathrm{I})$-hydride and styryl boronate ester, ${ }^{50}$ indicating the feasibility of two elementary steps of the proposed catalytic cycle (steps 1,2). In turn, (ii) $\mathrm{Rh}(\mathrm{I})$-hydrides are known to react selectively with alkenes to form $\mathrm{Rh}(\mathrm{I})$-alkyl species tolerating a broad range of functional groups (step 3). ${ }^{51-54}$ (iii) Although the final elementary step, the $\beta$-boryl elimination, (step 4), is unreported for Rhcomplexes, the principle of microscopic reversibility for the inverse step, i.e., an alkene insertion into a $\mathrm{Rh}(\mathrm{I})-\mathrm{B}$ bond, ${ }^{50,55}$ supported its feasibility. (iv) Lastly, the engagement of only lowvalent $\mathrm{Rh}(\mathrm{I})$-intermediates obviates otherwise impeding sidereactions based on reductive-elimination reactivity of high-valent complexes, such as competitive hydroboration or hydrogenation. The chemoselectivity of the alkene insertion into a $\mathrm{Rh}(\mathrm{I})-\mathrm{B}$ bond remained an open question. ${ }^{56-61}$ 
(a) Desired features for alkene borylation strategies

$$
\begin{gathered}
\text { Compatible with common functional groups? } \\
\text { Applicable to terminal \& internal (cyclic) alkenes? } \\
\text { Site-, regio-, and stereoselectivity? }
\end{gathered}
$$

(b) Borylation by well-established olefin cross-methathesis

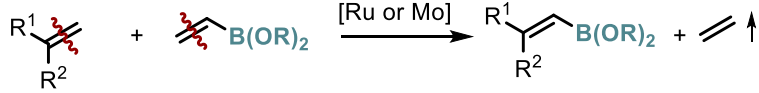

$$
\begin{aligned}
& \text { cleavage of carbon-carbon bond precludes borylation of } \\
& \text { internal (cyclic) alkenes without skeleton modification }
\end{aligned}
$$

(c) This study: boryl group transfer strategy under $\mathrm{Rh}(\mathrm{I})$-catalysis

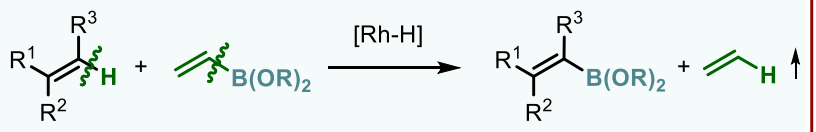

$\mathrm{R}=\mathrm{H}$, Alkyl, Ar, EWG, etc.

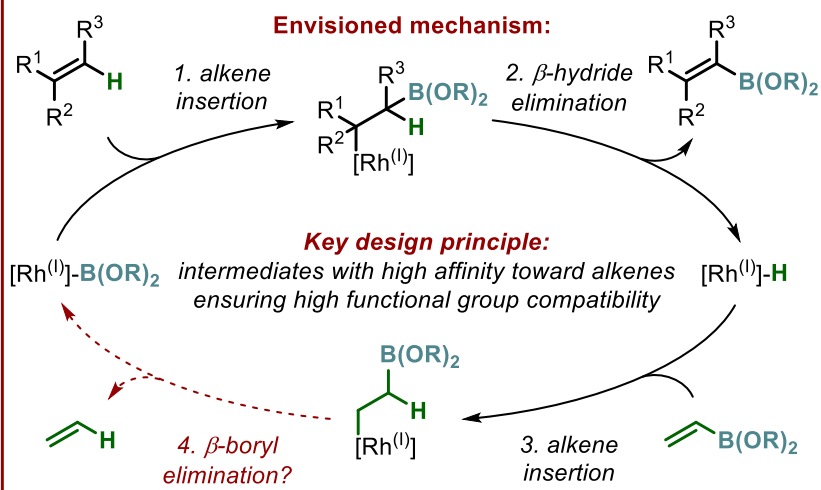

Key features of this study:

- Support for the mechanism with an unprecedented $\beta$-boryl elimination

- Detailed insight into the selectivity-controlling features

- Compatibility with common functional groups \& transfer of different boryl groups (e.g., alkyne, aldehyde, amine, pyridine \& Bpin, Bmac, Bpnd)

- Applicability to a variety of terminal \& internal alkenes confirmed

Figure 1. Context of this work. (a) $\mathrm{C}-\mathrm{H}$ borylation of alkenes with appealing features for fine-chemical synthesis, (b) state of the art - alkene cross-metathesis, (c) transfer borylation reaction under $\mathrm{Rh}(\mathrm{I})$-catalysis - summary of this work.

Here we report our studies establishing the feasibility, mechanism, and key features of transfer $\mathrm{C}-\mathrm{H}$ borylation of alkenes under Rh(I)-catalysis. A series of experimental mechanistic studies, corroborated by DFT calculations, provided insight into the details of the catalytic cycle that introduces the thus far unreported yet relatively fast $\beta$-boryl elimination step engaging the $\mathrm{Rh}(\mathrm{I})$-( $\beta$-borylalkyl) intermediate. The studies revealed the rate and selectivity determining aspects of the reaction. Further, the strategy proved to be applicable not only to terminal but also so far challenging internal alkene starting materials and compatible with a broad range of functional groups, including motifs that are typically problematic using established approaches, thus setting the stage for developing new valuable hydrogenfor-functional group exchange transformations for fine-chemical synthesis.

\section{RESULTS AND DISCUSSION}

Feasibility \& catalyst formulation for a model reaction.
We commenced our studies by evaluating the feasibility of the devised reactivity in a model reaction of styrene 1a with vinyl boronate pinacol ester $\mathbf{2}$ forming product 3a. To identify a suitable catalyst, we considered precursors that could form the prospective species that operate in the catalytic cycle, i.e., either a $\mathrm{Rh}(\mathrm{I})$-hydride or a $\mathrm{Rh}(\mathrm{I})$-boryl complex (Figure 1c). We selected phosphine-Rh(I)-alkoxide complexes, which were reported to form coordinatively unsaturated $\mathrm{Rh}(\mathrm{I})$-hydride species through migratory insertion of an olefin into the $\mathrm{Rh}-\mathrm{O}$ bond and subsequent $\beta$-hydride elimination. ${ }^{62,63}$

Upon evaluation of a range of complexes of different phosphine ligands and conditions (Table 1, Tables S1-S5), we found that the model reaction of $\mathbf{1 a}$ with $\mathbf{2}$ (1.5 equiv) in the presence of $[\mathrm{Rh}(\mathrm{cod}) \mathrm{OMe}]_{2}$ and xantphos formed product 3a in $92 \%$ yield (entry 1), confirming the successful reaction design. Importantly, no other isomers of the product were observed. It should be noted that, because this isodesmic reaction is merely exergonic $\left(\Delta \mathrm{G}^{\circ}=-0.57 \mathrm{kcal} / \mathrm{mol}\right),{ }^{64}$ the release of the gaseous ethene by-product is a driving force of the reaction. The formation of ethene was confirmed by in situ NMR spectroscopy. ${ }^{64}$

Table 1. Influence of reaction conditions.

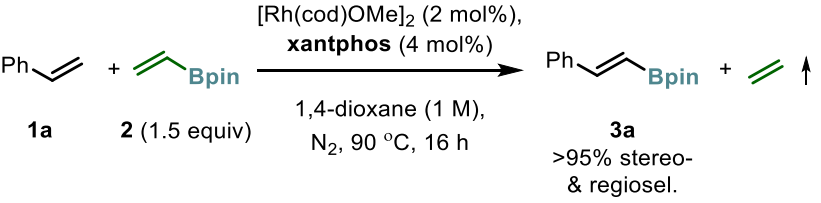

$$
\begin{aligned}
& \text { apephos } \\
& \begin{array}{ccc}
\text { entry variations from standard conditions } & \text { yield }(\%) \\
1 & & 92
\end{array} \\
& 2 \quad[\mathrm{Rh}(\operatorname{cod}) \mathrm{Cl}]_{2} \text { as precursor }^{\mathrm{b}} \quad<2 \\
& 3 \quad[\mathrm{Rh}(\mathrm{cod}) \mathrm{Cl}]_{2}+\mathrm{NaOMe} \text { as } \text { precursor }^{\mathrm{b}} \quad 92 \\
& 4 \quad[\mathrm{Rh}(\operatorname{cod}) \mathrm{Cl}]_{2}+\mathrm{NaOtBu} \text { as } \text { precursor }^{\mathrm{b}} \quad 92 \\
& 5 \quad \text { no }[\mathrm{Rh}(\mathrm{cod}) \mathrm{OMe}]_{2}<2 \\
& 6 \text { no xantphos } 18 \\
& 7 \text { dpephos instead of xantphos }<2
\end{aligned}
$$

${ }^{a}$ Yield determined by ${ }^{1} \mathrm{H}$ NMR analysis of the reaction mixture with an internal standard; ${ }^{\mathrm{b}}$ instead of $[\mathrm{Rh}(\mathrm{cod}) \mathrm{OMe}]_{2} ; 1: 1$ ratio of $\mathrm{Rh}$ to sodium alkoxide. For reactions with other phosphine ligands and reaction conditions, see section 2 of the SI.

The phosphine-Rh(I)-alkoxide complex is key to the catalytic activity, as confirmed in a series of control experiments (Table $1)$. No reaction was observed with $[\mathrm{Rh}(\operatorname{cod}) \mathrm{Cl}]_{2}$ in place of $[\mathrm{Rh}(\mathrm{cod}) \mathrm{OMe}]_{2}$ (entry 2). However, the catalytic activity was recovered when $[\mathrm{Rh}(\operatorname{cod}) \mathrm{Cl}]_{2}$ was used with either sodium methoxide or sodium tert-butoxide (entries 3-4) that formed the rhodium(I)-alkoxide complexes in situ. No reaction occurred in the absence of the rhodium precursor (entry 5), but low activity of the rhodium(I)-alkoxide precursor was maintained in the absence of any phosphine ligand (18\% yield, entry 6$)$.

\section{Mechanistic Studies.}

Having established the feasibility of the boryl group transfer between alkenes under phosphine-Rh(I)-alkoxide catalysis, 
open questions arose regarding the mechanistic aspects of the reaction. In particular, we wondered whether the reaction occurred in the proposed catalytic cycle involving $\mathrm{Rh}(\mathrm{I})$-hydride and $\mathrm{Rh}(\mathrm{I})$-boryl intermediates with the $\mathrm{Rh}(\mathrm{I})-(\beta$-borylalkyl) intermediates engaging in the so far unreported $\beta$-boryl elimination step (see Figure 1c) or whether an alternative mechanism involving a $\mathrm{Rh}$-alkoxide mediated $\mathrm{C}\left(\mathrm{sp}^{2}\right)-\mathrm{H}$ bond activation was operating. ${ }^{65-67}$ The origin of the excellent regio- and stereoselectivity remained to be solved as well. In addition, it was uncertain whether the activation of the precatalyst would undergo as initially hypothesized. To shed light onto the above questions, we performed a series of experimental studies, which we further corroborated by DFT calculations.

Probing Alternative Catalytic Cycles. Stereochemical considerations of different plausible catalytic cycles implied different fate of the olefinic hydrogen atoms of alkene 1a depending on the mechanism of the reaction (paths I-II, Figure 2a). To differentiate between these scenarios, we performed catalytic experiments with deuterium-labeled starting materials (Figure $2 b)$. The reaction of $(E)-\beta$-deuteriostyrene $1 \mathbf{a}-\beta-d$ furnished product $3 \mathbf{a}$ that partially retained deuterium labeling (reaction
1). This outcome of 3a maintaining some deuterium is inconsistent with the mechanism involving a $\mathrm{C}-\mathrm{H}$ bond activation by the $\mathrm{Rh}(\mathrm{I})$-alkoxide species (path II, Figure 2a), but it is consistent with the mechanism involving the alkene insertion into the $\mathrm{Rh}(\mathrm{I})-\mathrm{Bpin}$ followed with the $\beta$-hydride elimination to form the product (path I). Further, both $(E)$ - or $(Z)$ - $\beta$-deuteriostyrene 1a- $\beta-d$ reacted with $\mathbf{2}$ forming product $\mathbf{3 a}$ with similar content and relative distribution of deuterium at the $\alpha$ - and $\beta$-sites (reactions 1-2). These outcomes suggest that the alkene starting materials also insert reversibly into the $\mathrm{Rh}-\mathrm{H}$ bond leading to the deuterium scrambling between different sites of alkenes (paths III-IV). In accordance, in situ NMR spectroscopy and GC-MS analysis of the reaction of styrene- $d_{8} \mathbf{1 a}-d_{8}$ with 2 revealed the extensive hydrogen/deuterium scrambling not only throughout the $\alpha$ - and $\beta$-sites of product 3a but also between both alkene starting materials $\mathbf{1 a}-d_{8}$ and $\mathbf{2}$ occurring already early in the process (reactions 3-4). This result indicates that the reversible insertions into the $\mathrm{Rh}-\mathrm{H} / \mathrm{Rh}-\mathrm{D}$ bonds are faster than the transfer of the boryl group. We also found that the product re-enters the cycle during the catalytic reaction. Compound $\mathbf{3 b}$,

(a) Implications of alkene insertion \& $\beta$-elimination sequence or alternative $\mathbf{C}-\mathrm{H}$ bond activation mechanism

path I. insertion into a $R$ h-B bond - $\beta-H$ elim. removes $H^{Z}$ \& inverts $H^{E}$ to the (Z)-side path III. reversible alkene insertion into a Rh-H scrambles $H^{Z}$ \& $H^{E}$

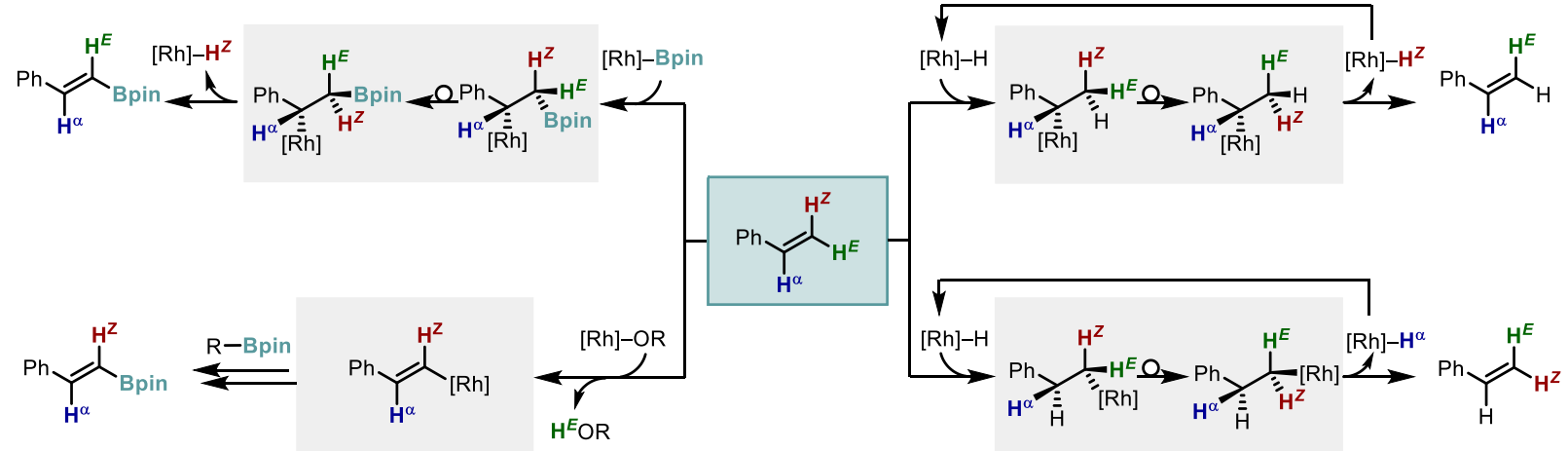

path II. $\mathrm{C}-\mathrm{H}$ bond activation removes $\mathrm{H}^{E}$ \& retains $\mathrm{H}^{\mathrm{Z}}$ at the (Z)-side

path IV. reversible inverted alkene insertion into a $\mathrm{Rh}-\mathrm{H}$ scrambles $\mathrm{H}^{\alpha}$

(b) Experiments with isotope-labeled starting materials probing the mechanism of the reaction

(1)

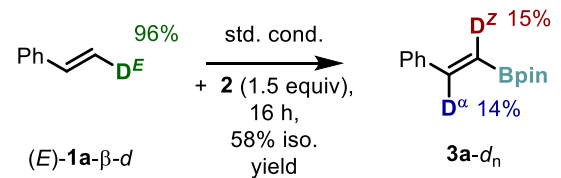

(2)

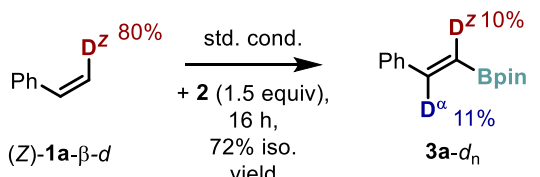

(3)

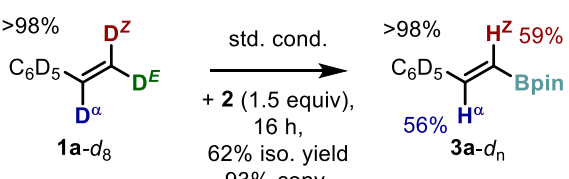

(4)<smiles>[2H]/C([18OH])=C(/[2H])[PH3+]</smiles>

(5)

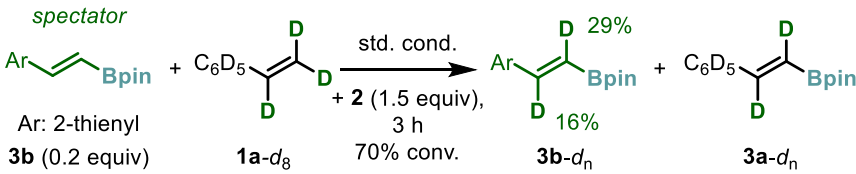

(6)

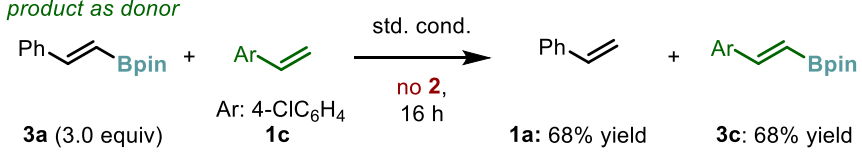

Figure 2. Mechanistic experiments probing the prospective catalytic cycle for the boryl group transfer. (1-2): Partial deuterium retaining in 3a in the reaction of $(E)-\mathbf{1 a}-\beta-d$ is consistent with the mechanism involving alkene insertion into a Rh-B bond $\& \beta$-hydride elimination sequence; (1-4): H/D scrambling between starting materials indicates fast \& reversible alkene insertion into the $\mathrm{Rh}-\mathrm{H}$ intermediate; (5): Deuterium incorporation into $\mathbf{3 b}$ indicates that the product re-enters the cycle; (6): Boryl group transfer between 3a and 1c indicates that the reaction is reversible. For standard conditions, see Table 1; for further details, see the SI. 
the analogue of 3a bearing a different aryl ring, added to the reaction of $\mathbf{1 a}-d_{8}$ and $\mathbf{2}$ as a 'spectator' underwent partial deuterium incorporation into its $\alpha$ - and $\beta$-positions, implying its reversible insertion into the $\mathrm{Rh}-\mathrm{H} / \mathrm{Rh}-\mathrm{D}$ bond occurring also early in the process ( $3 \mathrm{~h}$; reaction 5). Lastly, utilizing compound 3a as the boryl group donor instead of vinyl boronate $\mathbf{2}$ in the reaction of 4-chlorostyrene 1c, we observed the formation of $\mathbf{3 c}$, 4-chloro analogue of $\mathbf{3 a}$ and styrene $\mathbf{1 a}$ (reaction 6), indicating the reversibility of all steps of the cycle.

Insight into the Rate Limiting Step. With the observed fast hydrogen/deuterium scrambling (Figure 2b), we considered that both the insertion of alkenes into the $\mathrm{Rh}(\mathrm{I})$-hydride and $\beta$-hydride elimination must be fast, and hence the overall rate of the reaction is limited by either the alkene insertion into the $\mathrm{Rh}(\mathrm{I})$ boryl intermediate or the $\beta$-boryl elimination step (Figure 1c). To differentiate between these scenarios, we studied the reactivity of the independently prepared $\mathrm{Rh}(\mathrm{I})$-boryl intermediate, LRhBpin (Figure 3). ${ }^{64,68}$ First, we found that LRhBpin reacted with alkene 1a to form product 3a gradually in time, implying a rather slow alkene insertion into the $\mathrm{Rh}-\mathrm{B}$ bond (Figure $3 \mathrm{a}$ ). In turn, we observed that $\mathbf{L R h B p i n}$ is catalytically active in the model reaction, with similar rates of the formation of $\mathbf{3 a}$ early in the reaction, i.e., before the first catalytic turnover, and later in the reaction, i.e., after the first catalytic turnover (Figure 3b). Most importantly, the formation of $\mathbf{3 a}$ before the first turnover involves only the insertion of alkene into the $\mathrm{Rh}-\mathrm{B}$ bond of starting LRhBpin (and the fast $\beta$-hydride elimination), while the formation of $\mathbf{3 a}$ in the later phase of the reaction requires all steps of the cycle, including the $\beta$-boryl elimination step. Thus, similar rates in different phases of the reaction indicate that the alkene insertion into the $\mathrm{Rh}-\mathrm{B}$ bond is slower than the $\beta$-boryl elimination, and hence is most likely rate-limiting of the catalytic process.

Probing Stereo- and Regiocontrol of the Reaction. Because product $\mathbf{3 a}$ is more stable than isomers $(Z)-\mathbf{3 a}$ and $(\alpha)-\mathbf{3 a}$ $(0.0$ vs. +4.2 and $+5.0 \mathrm{kcal} / \mathrm{mol}$, respectively $),{ }^{64}$ the stereo- and regioselectivity of the reaction might be controlled either thermodynamically or kinetically. To differentiate between these scenarios, we studied the reactivity of independently prepared isomers ( $Z$ )-3a and ( $\alpha)$-3a under catalytic conditions. We observed that stereoisomer (Z)-3a underwent fast conversion to $(E)$-isomer 3a, when present as a spectator of a catalytic reaction of substrate $\mathbf{1 d}$ under otherwise standard conditions (Figure 4 , reaction 1). This indicates that stereoselectivity might reflect the relative stability of the products. However, as the interconversion occurs most likely through the fast insertion of highenergy ( $Z$ )-3a into the catalytic $\mathrm{Rh}(\mathrm{I})$-hydride intermediate followed by $\beta$-hydride elimination, the data cannot rule out that stereoselectivity is under kinetic control at the actual $\beta$-hydride elimination step. In contrast, in an analogous experiment, the $\alpha$ regioisomer of 3a, $(\alpha)$-3a was rather reluctant to the isomerization: only $11 \%$ of $(\alpha)$-3a converted to $\mathbf{3 a}$ after $3 \mathrm{~h}$, while at the same time the (completely $\beta$-selective) catalytic reaction was nearly finished ( $84 \%$ yield of $\mathbf{3 e}$; Figure 4 , reaction 2 ). Overall, the conversions of $(Z)-\mathbf{3 a}$ and $(\alpha)-\mathbf{3 a}$ to $\mathbf{3 a}$ indicate that both prospective products of the reaction can enter the catalytic cycle, and hence, due to the principle of microscopic reversibility, they could be formed in the reaction as well. However, slow conversion of the high-energy isomer $(\alpha)$-3a to $\mathbf{3 a}$, which is slower than the actual catalytic reaction, indicates that the regioselectivity of the reaction is controlled kinetically, a rather unusual feature in functional group transfer catalysis. ${ }^{69}$ Most importantly, because the kinetic control of the selectivity depends on the catalyst, the data suggest the feasibility of accessing different isomers using different catalysts.

(a) Reactivity of $\mathrm{Rh}(\mathrm{I})$-Bpin intermediate with an alkene

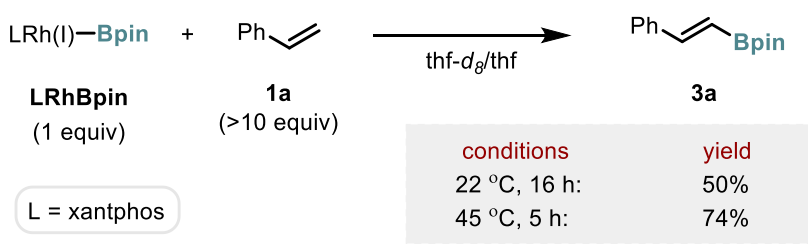

(b) Catalytic activity of $\mathrm{Rh}(\mathrm{I})-\mathrm{Bpin}$ intermediate

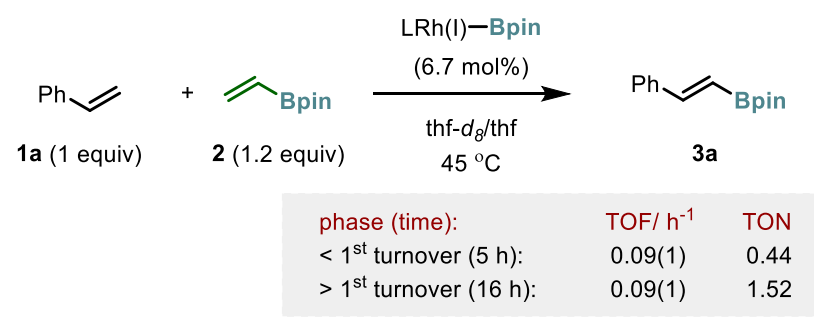

Figure 3. Reactivity of independently prepared xantphos$\mathbf{R h}(\mathbf{I})$-Bpin intermediate. $\mathbf{L R h B}$ pin reacts with 1 a rather slowly and is catalytically active under similar conditions; the formation of $\mathbf{3 a}$ with similar rates in the different phases of the catalytic reaction suggests that the alkene insertion is rate-liming. TOF, turnover frequency; TON, turnover number; for full experimental details, see section 3.8 of the SI.

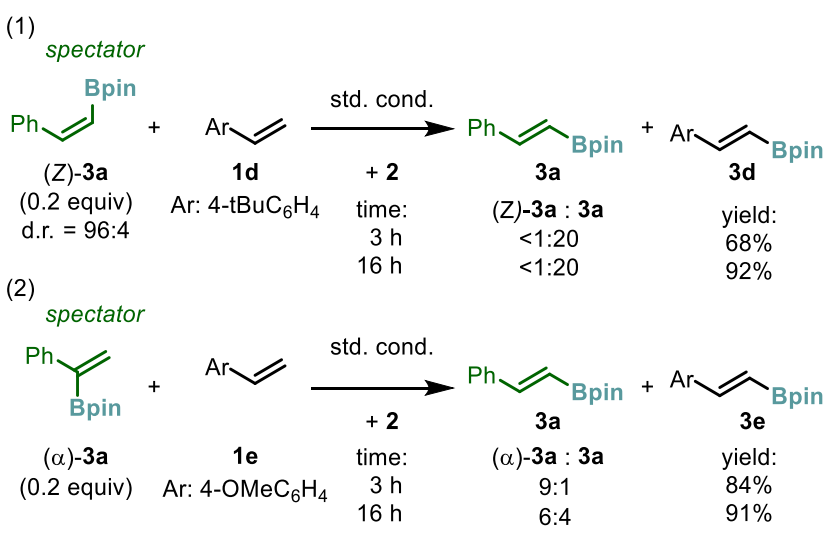

Figure 4. Mechanistic experiments probing the pathways toward formation of stereo- and regioisomeric products. (1): Fast isomerization of $(Z)-\mathbf{3 a}$ to $(E)-\mathbf{3 a}$ indicates that the stereoselectivity of the reaction might be thermodynamically controlled or kinetically controlled at the $\beta$-hydride elimination step. (2) As the isomerization of $(\alpha)-\mathbf{3 a}$ to $\mathbf{3} \mathbf{a}$ is slower than the catalytic reaction, the regioselectivity is controlled kinetically. For standard conditions, see Table 1; for details, see section 3.2 of the SI.

Precatalyst Activation with an Alkene. Our NMR experiments indicated that the $\mathrm{Rh}(\mathrm{I})$-alkoxide undergoes an intermolecular insertion of an alkene and $\beta$-hydride elimination to form $\mathrm{Rh}(\mathrm{I})$-hydride species (Scheme 1), albeit the process is not quantitative. Specifically, as judged by the characteristic hydride signal at $-12.4 \mathrm{ppm}$ in the ${ }^{1} \mathrm{H}$ NMR spectrum, up to $16 \%$ of a Rh-hydride species was formed after $2 \mathrm{~h}$ at $22{ }^{\circ} \mathrm{C}$ in the stoichiometric reactions of alkene 1a with xantphos-Rh(I)alkoxide containing either the $\mathrm{MeO}$ or $\mathrm{tBuO}$ ligand (see section 3.6 of the SI). It is worth noting that these reactions were 
performed in the presence of $\mathrm{PCy}_{3}$ as an auxiliary ligand that stabilizes the hydride complex, but its presence is not essential for the hydride formation. ${ }^{63}$ Additionally, no increase of the yield was observed upon further reaction at this or elevated temperatures, but a mixture of other unidentified complexes was formed as judged by ${ }^{31} \mathrm{P}$ NMR spectroscopy. ${ }^{64}$ In turn, no hydride species was formed in the experiment with xantphos$\mathrm{Rh}(\mathrm{I})-\mathrm{OMe}$ in the absence of an alkene, excluding an alternative mechanism of the formation of $\mathrm{Rh}-\mathrm{H}$ species through the direct $\beta$-hydride elimination from the methoxy ligand.

Scheme 1. Precatalyst activation through an alkene insertion into a Rh-O bond and $\beta$-hydride elimination.

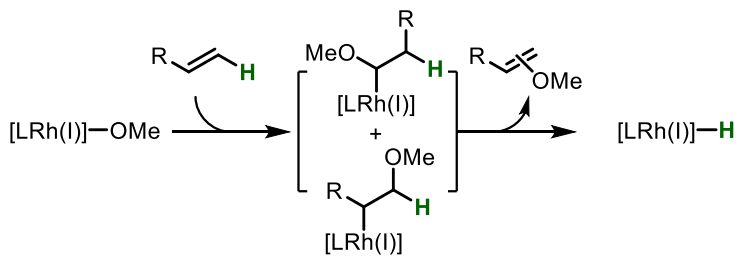

Alternative Alkene-Independent Activation of the Precatalyst. Other efficient strategies for precatalyst activation that do not depend on alkene starting materials could be useful, especially when these are less prone to undergo migratory insertion into a $\mathrm{Rh}(\mathrm{I})-\mathrm{O}$ bond, e.g., internal alkenes. Because a rhodium(I)alkoxide complex reacts readily with $\mathrm{B}_{2} \mathrm{pin}_{2}$ to form a $\mathrm{Rh}(\mathrm{I})$ Bpin complex, ${ }^{68}$ the other catalytic intermediate of the cycle (cf. Figure 1c), we tested the influence of $\mathrm{B}_{2} \mathrm{pin}_{2}$ on the reaction.

\section{Scheme 2. Activation through a reaction with $B_{2} p_{2}$.}

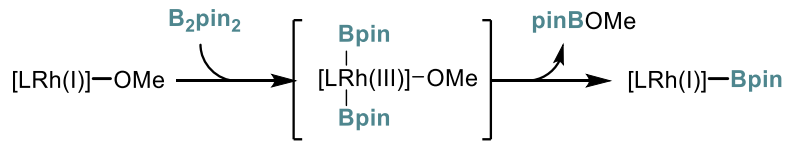

The presence of co-catalytic $\mathrm{B}_{2} \mathrm{pin}_{2}$ is beneficial for the activity, as observed by the comparison of the model reaction in its presence or absence (Figure 5). Following both reactions in

(a) Precatalyst activation with an alkene
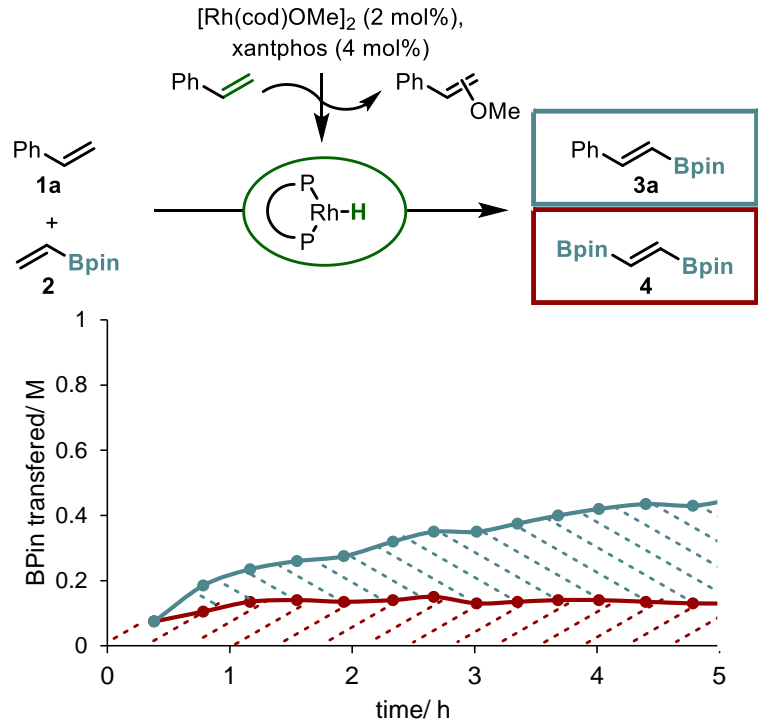

time using in situ ${ }^{1} \mathrm{H}$ NMR spectroscopy revealed that the reaction in the presence of small amounts of $\mathrm{B}_{2} \mathrm{pin}_{2}$ occurred at over 2 -fold higher rate than the analogous reaction in its absence. Most likely, the reason for the increased rate is the higher level of precatalyst activation by $\mathrm{B}_{2} \mathrm{pin}_{2}$. Interestingly, in the initial phase of the reaction, the boryl group transfer occurred largely to form vinyl $(E)$-1,2-bisboronate $\mathbf{4}$, which was followed by the phase of the formation of product $\mathbf{3 a}$ and the consumption of $\mathbf{4}$ (Figure 5). Most importantly, the protocol with co-catalytic $\mathrm{B}_{2} \mathrm{pin}_{2}$ additive enables to lower the catalyst loading substantially (Table $\mathrm{S} 7$ ). While reactions without $\mathrm{B}_{2} \mathrm{pin}_{2}$ required 2 $\mathrm{mol} \%$ of $[\mathrm{Rh}(\mathrm{cod}) \mathrm{OMe}]_{2}$, those with small amounts of $\mathrm{B}_{2} \mathrm{pin}_{2}$ furnished the product in similar yields with up to 8-fold lower loading (as low as $0.24 \mathrm{~mol} \%$ ). The enabling feature of $\mathrm{B}_{2} \mathrm{pin}_{2}$ is further manifested in reactions of more challenging substrates, such as internal alkenes, in case of which nearly no activity is observed in the absence of co-catalytic $\mathrm{B}_{2} \mathrm{pin}_{2}$ (vide infra).

Density Functional Theory (DFT) Studies. The experimental mechanistic findings were corroborated by DFT calculations performed at the M06-L/def2-TZVP ${ }_{\text {(SMD,1,4-dioxane) }} / / \mathrm{M} 06-\mathrm{L} / \mathrm{def} 2-$ SVP level of theory. The computed free energy surfaces for the formation of 3a, $(Z)-\mathbf{3 a}$ and $(\alpha)$-3a (Figure 6 ) demonstrated the feasibility of the initially devised catalytic cycle (Figure 1c) and further supported the alkene insertion into the $\mathrm{Rh}-\mathrm{B}$ bond as the rate-limiting step of the reaction. Importantly, our computations not only support that regioselectivity is kinetically controlled but also revealed that stereoselectivity is also under kinetic control. Additionally, we provided a detailed analysis on the unprecedented $\beta$-boryl elimination process (section 4.3 of the SI), as well as an insight into the preferential P-P coordination mode of xantphos during the reaction (section 4.2 of the SI).

Specifically, the initial xantphos-Rh(I)-hydride complex coordinated by boryl group donor $\mathbf{2}, \mathbf{L R h H}\left(\eta^{2}-2\right)$ is predicted to readily form the $\mathrm{Rh}(\mathrm{I})$-boryl intermediate, LRhBpin $\left(\eta^{2}\right.$-ethene $)$ in an exergonic process $\left(\Delta \mathrm{G}_{\mathrm{rel}}=-6.0 \mathrm{kcal} / \mathrm{mol}\right)$ through an alkene insertion (via transition state 1, TS-1) - $\beta$-boryl elimina-

(b) Precatalyst activation with $\mathrm{B}_{2} \mathrm{pin}_{2}$

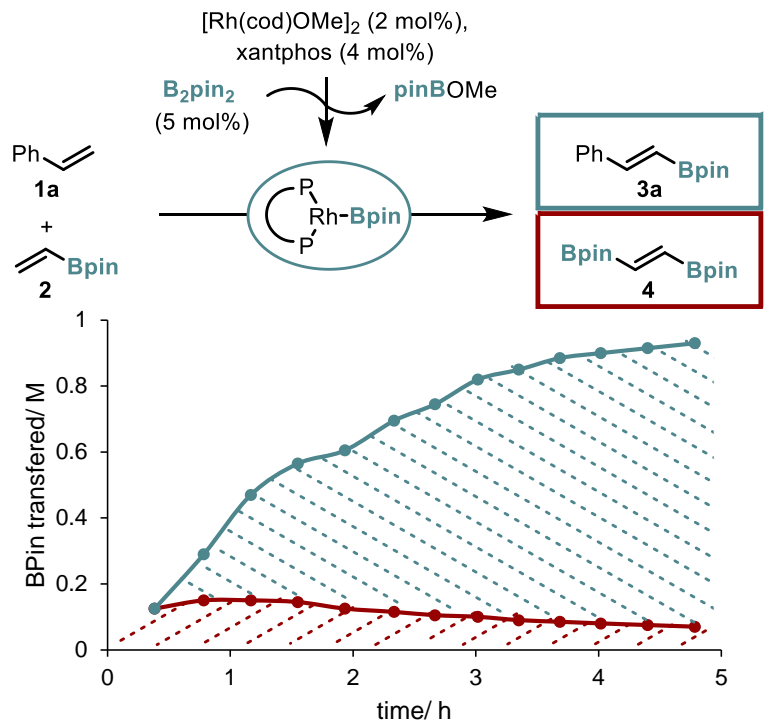

Figure 5. Evaluation of an alternative precatalyst activation - influence of catalytic amounts of $\mathbf{B}_{\mathbf{2}}$ pin 2 . Reactions at $76^{\circ} \mathrm{C}$ in $1,4-$ dioxane/ $\mathrm{C}_{6} \mathrm{D}_{6}(87 / 13 \mathrm{v} / \mathrm{v})$ in J-Young NMR tubes and monitored in situ by ${ }^{1} \mathrm{H}$ NMR spectroscopy; for details, see the SI. 


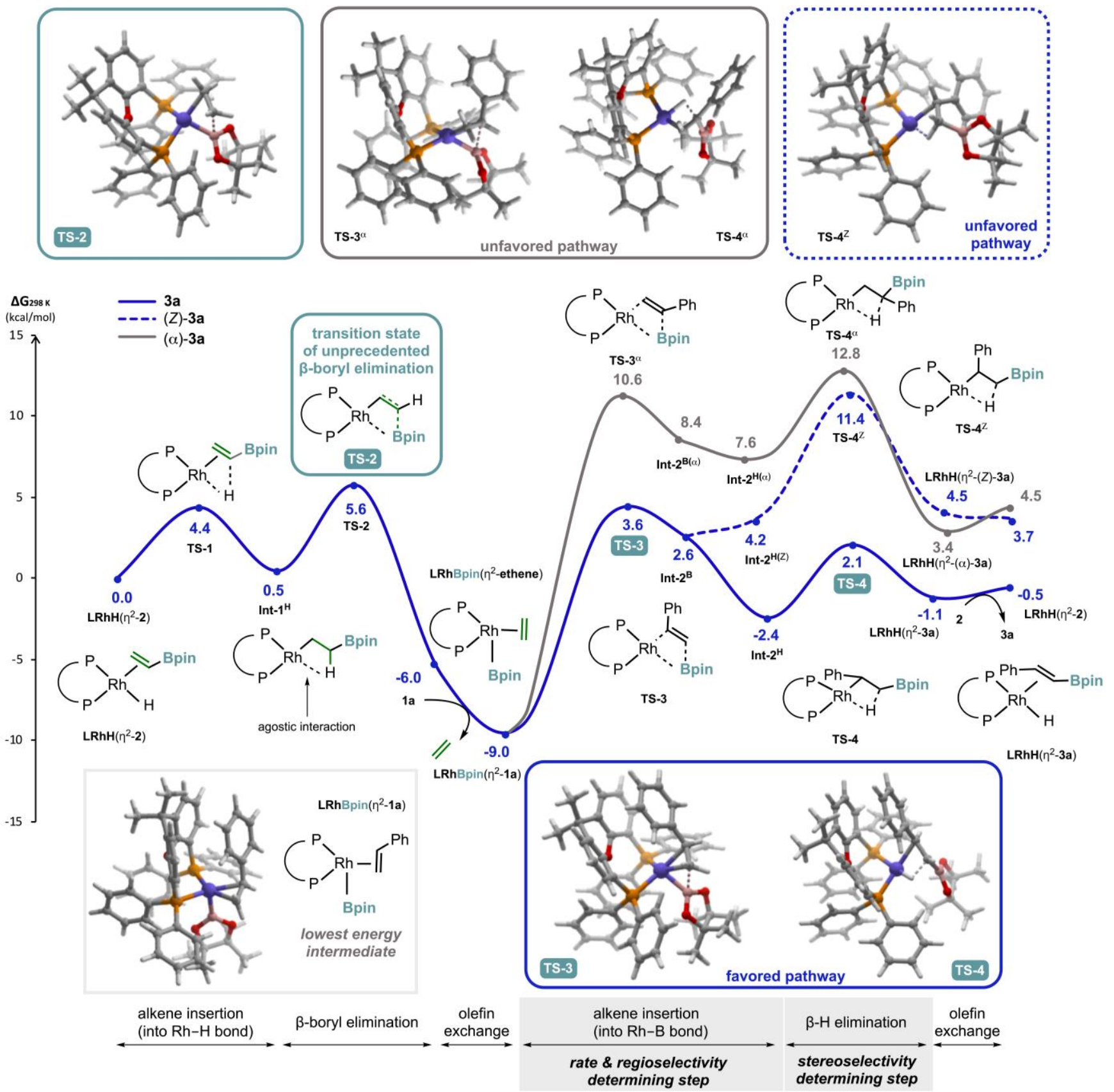

Figure 6. DFT investigation of the free energy surfaces for the reaction pathways leading to products 3a, $(Z)-3 a$, or $(\alpha)-3 a$. Calculations have been performed at the M06-L/def2-TZVP(SMD)//M06-L/def2-SVP level of theory in 1,4-dioxane as solvent (SMD solvation model); SMD, solvation model based on density; Int, intermediate, TS, transition state. For full discussions, structures of all transition states and intermediates, and further details, see the SI.

tion (TS-2) sequence with a low free energy barrier of +5.6 $\mathrm{kcal} / \mathrm{mol}$. Noteworthy, the oxygen atom of the xantphos ligand does not interact with the metal center during the process, while such an interaction was found to be occurring in other POP-Rhpromoted reactions. ${ }^{61}$ Parallel calculations indicate that such a pathway would lead to a significantly higher free energy barrier $\left(\Delta \mathrm{G}_{\mathrm{rel}}=+10.5 \mathrm{kcal} / \mathrm{mol} \mathrm{vs}+5.6 \mathrm{kcal} / \mathrm{mol}\right.$; see section 4.2 of the SI). Therefore, the activity of the xantphos-Rh(I) complex originates most likely from the distinct wide bite angle of the ligand rather than the presence of the oxygen atom. In accordance, no activity was observed for the analogous complex bearing flexible dpephos (cf. Table 1).
The borylation of 1a with the thus-formed LRhBpin $\left(\eta^{2}\right.$-ethene) intermediate embarks upon a ligand exchange to form LRhBpin $\left(\eta^{2}-1\right.$ a) (along with the extrusion of ethene), which is the lowest energy intermediate of the catalytic cycle $\left(\Delta \mathrm{G}_{\mathrm{rel}}=\right.$ $-9.0 \mathrm{kcal} / \mathrm{mol}$; see section 4.4 of the SI for full discussion on olefin exchange processes). The subsequent alkene insertion into its $\mathrm{Rh}(\mathrm{I})-\mathrm{B}$ bond is predicted to be rate-limiting with the free energy barrier of $+12.6 \mathrm{kcal} / \mathrm{mol}$ (TS-3). The following $\beta$ hydride elimination to form product 3a through TS-4 proceeds with a low barrier $(+4.5 \mathrm{kcal} / \mathrm{mol})$. Product 3a is released from $\operatorname{LRhH}\left(\eta^{2}-\mathbf{3 a}\right)$ upon the ligand exchange with another molecule of 2 , closing the catalytic cycle. Notably, the alternative $\beta$-hydride elimination step toward the formation of stereoisomeric 
product ( $Z$ )-3a involves a significantly higher energy barrier (TS-4 ${ }^{Z}$ ), even greater than that for the alkene insertion into the $\mathrm{Rh}-\mathrm{B}$ bond (TS-3), accounting for the overall barrier of +20.4 $\mathrm{kcal} / \mathrm{mol}$ (from LRhBpin $\left(\eta^{2} \mathbf{- 1 a}\right)$; dotted blue line in Figure 6). Further, the reaction pathway toward the formation of regioisomeric product $(\alpha)$-3a involves higher energy transition states for both alkene insertion (TS-3 ${ }^{\alpha}$ ) and $\beta$-hydride elimination $\left(\right.$ TS- ${ }^{\alpha}$ ) with the overall barrier of $+21.8 \mathrm{kcal} / \mathrm{mol}$ (grey line in Figure 6). Noteworthy, computations also predict that both $(Z)$ 3a and $(\alpha)$-3a can enter the catalytic cycle with energy barriers of +6.9 and $+9.4 \mathrm{kcal} / \mathrm{mol}$, respectively. These results correlate well with the observed interconversions of $(Z)-\mathbf{3 a}$ and $(\alpha)-\mathbf{3 a}$ to product 3a, which were found to be fast and slow in comparison to the rate of the actual catalytic reaction, respectively (cf. Figure 4).

It is worth mentioning that a stepwise analysis of the $\beta$-boryl elimination process revealed that prior to the transition state, there is an intermediate engaging an interaction between boron and $\mathrm{Rh}$ atoms, which leads to the $\mathrm{C}-\mathrm{B}$ bond weakening and its elongation prior to the bond breaking event, and hence facilitating this unprecedented elementary reaction (see section 4.3 of the SI). For full discussions, including all higher-energy pathways, further details, and structures of all transition states and intermediates, see section 4 of the SI.

Summarized Mechanistic Proposal. Based on the collected data we propose that $\mathrm{Rh}(\mathrm{I})$-catalyzed transfer borylation of alkenes occurs as shown in Figure 7. The key mechanistic features are:

1. The $\mathrm{Rh}(\mathrm{I})$-alkoxide precatalyst enters the catalytic cycle either through the alkene insertion into the $\mathrm{Rh}-\mathrm{O}$ bond $-\beta$-hydride elimination sequence forming the catalytic Rh-hydride species (path a) or through its reaction with co-catalytic $\mathrm{B}_{2}$ pin $_{2}$ additive to form the catalytic $\mathrm{Rh}(\mathrm{I})$-boryl complex along with pinBOMe under $\mathrm{B}_{2}$ pin $_{2}$-based activation (path $b$ ).

2. In the catalytic cycle, the $\mathrm{Rh}(\mathrm{I})$-hydride intermediate coordinating to Bpin-donor 2, $\mathbf{L R h H}\left(\eta^{2}-\mathbf{2}\right)$, reacts readily to form the $\mathrm{Rh}(\mathrm{I})$-boryl species, $\mathbf{L R h B p i n}\left(\eta^{2}\right.$. ethene), through an alkene insertion into the $\mathrm{Rh}-\mathrm{H}$ bond - $\beta$-boryl elimination sequence (steps I-II).

3. LRhBpin $\left(\eta^{2}-1\right)$, formed upon olefin exchange of ethene for starting material $\mathbf{1}$, is the lowest energy intermediate of the catalytic cycle (step III), which is in equilibrium engaging other alkenes in the mixture (step III'). The complex undergoes rate-limiting regioselective alkene insertion (step IV), followed by the fast stereoselective $\beta$-hydride elimination (step V), and the release of product 3 through an olefin exchange, which completes the cycle (step VI).

4. The relatively low free energy barriers for all steps of the catalytic cycle have two main consequences: First, many elementary steps are reversible, which can lead to isomerization and scrambling processes (through steps I' and IV'). Second, the free energy surface for the overall transformation is relatively flat, which is essential for effective catalysis in isodesmic processes with a limited energetic driving force. However, the study shows that a catalyst might impose high energy barriers (e.g., competitive steps IV' or V') for the formation of different isomers of the products of similar energy, creating the prospects for the kinetic control of selectivity in functional group transfer catalysis. ${ }^{69}$

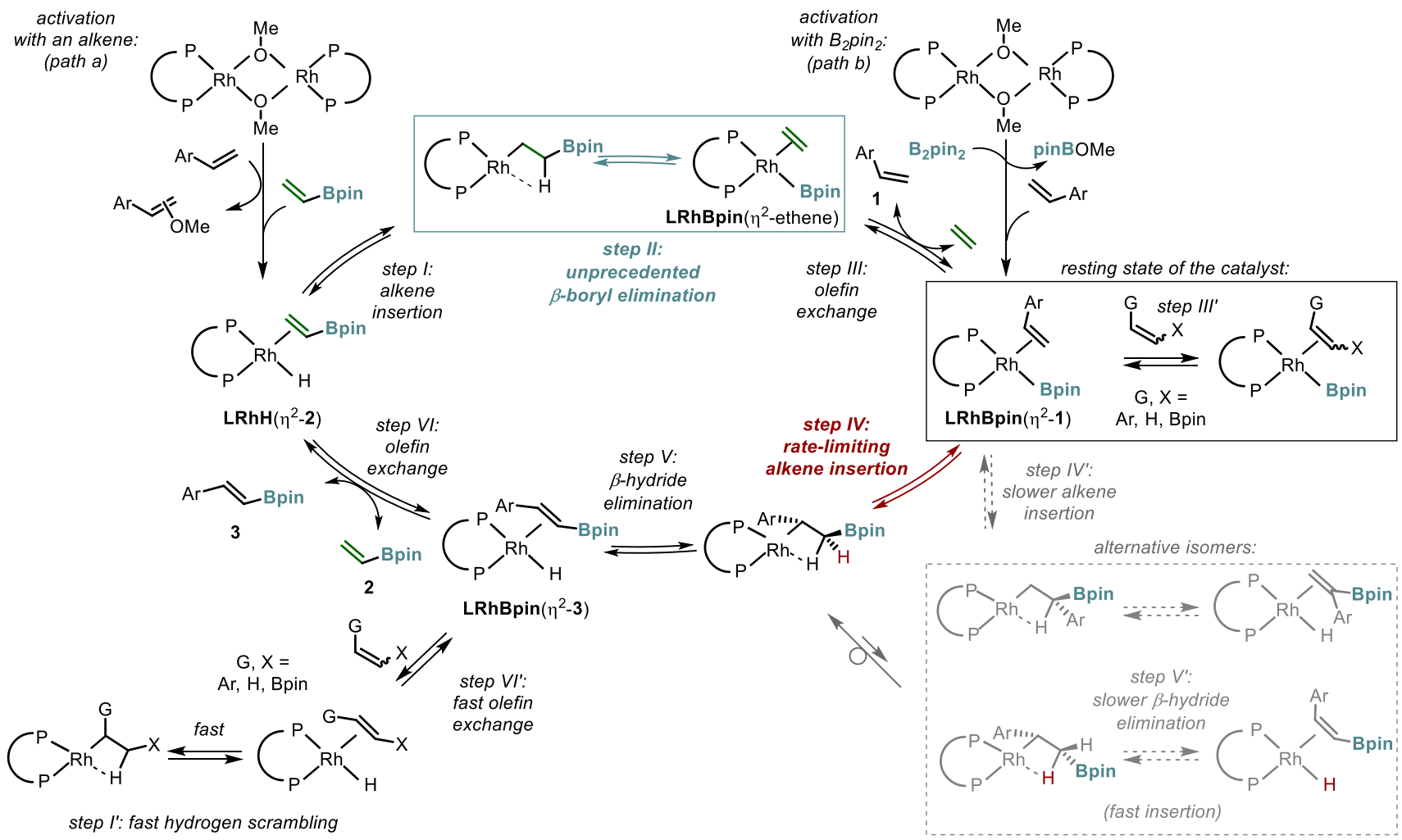

Figure 7. Full proposal of the mechanism of $\mathrm{Rh}(\mathrm{I})$-catalyzed transfer borylation of alkenes. 


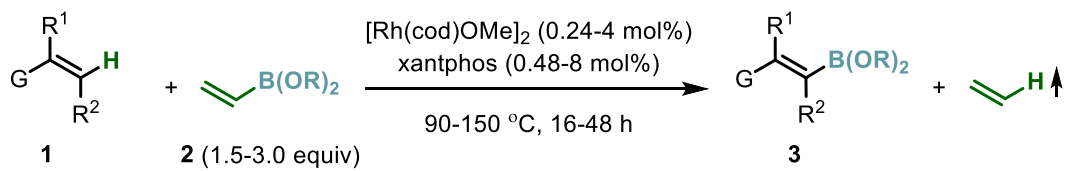

Diverse functional groups:

Heteroaromatic groups:<smiles>Fc1ccc(/C=C/[B]c2ccccc2)cc1</smiles>

$F G$ :

3f: $4-\mathrm{CHO}$

3g: $\quad 4-\mathrm{NMe}_{2}$

3h: $4-\mathrm{N}(\mathrm{Boc}) \mathrm{Me}$

3i: $\quad 4-\mathrm{COMe}$

3c: $\quad 4-\mathrm{Cl}$

3j: $\quad 4-\mathrm{CF}_{3}$

k: 3,5-bisCF

I: $4-\mathrm{F}$

3m: $\quad 4-\mathrm{CN}$

3n: $\quad 4-\mathrm{CO}_{2} \mathrm{Me}$

3o: 4-OAC

3e: $4-\mathrm{OMe}$

3p: $2-\mathrm{Me}$

3d: $4-\mathrm{tBu}$

$5 \mathrm{mmol}$ scale for $3 \mathrm{~d}: 76 \%$

with $0.24 \mathrm{~mol} \%$ of $[\mathrm{Rh}]$

$80 \%$<smiles>[B]/C=C/c1ccc(/C=C/c2ccc(Br)cc2)cc1</smiles>

$\mathrm{R}^{3}$

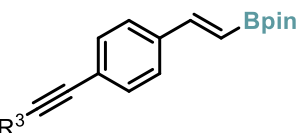

$3 r:^{b} R^{3}=t B u, \quad 53 \%$

$3 \mathrm{~s} \cdot \mathrm{b} \mathrm{R}^{3}=\mathrm{TMS}, 42 \%$

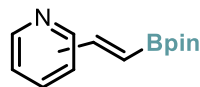

3t: $2-P y: 76 \%$

3u: 3-Py: $80 \%$

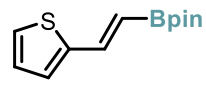

3b: $88 \%$

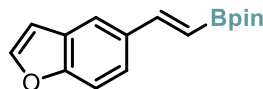

3v: $98 \%$

$\mathrm{C}_{6} \mathrm{H}_{13}$<smiles>C#CCc1ccc(C=C)cc1</smiles>
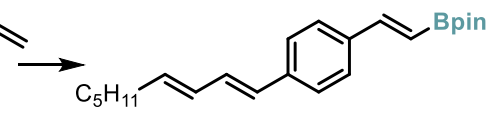

$1 w$

3w: $55 \%$

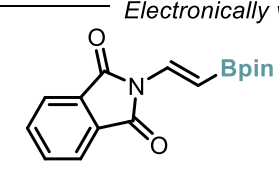

$3 \mathbf{x}: 80 \%$
$\mathrm{EtO}_{2} \mathrm{C} \overbrace{}^{\mathrm{Bpin}}$

3y: $54 \%$

Different substitution patterns \& congested double bonds:

Different boronic esters:
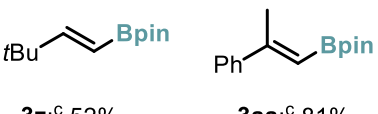

3aa:c $81 \%$

(90:10 dr)

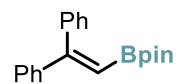

$3 \mathbf{a b}:^{\mathrm{c}} 88 \%$

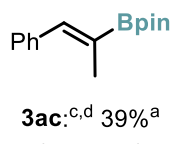

$(83: 17 \mathrm{dr})$

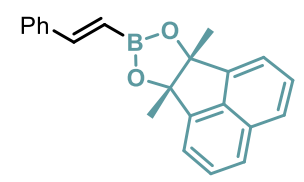

3ad: $86 \%$

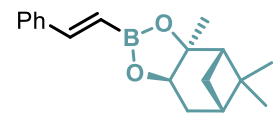

3ae: $92 \%$

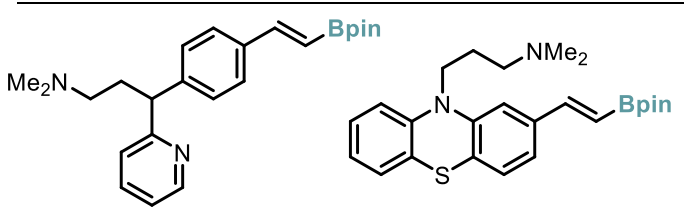

3af : Brompheniramine deriv. $76 \%^{a}$
$80 \%$<smiles>[B]/C=C/c1ccc(OC)c(-c2cc(C)c(C(=O)O)cc2C(C)(C)C)c1</smiles>

3ah: CD3254 deriv. $89 \%$<smiles>OB(O)/C=C/c1ccc(Cn2ccnc2)cc1</smiles>

5: Ozagrel bioisostere ${ }^{\mathrm{e}}$ (3ai: Bpin ester: $85 \%$ )<smiles>COc1cc(O)c(C(=O)OC(C)CCCC(C)OC(C)=O)c(O)c1</smiles>

3aj: ${ }^{f}$ Zearalenol deriv. $11 \%$

Figure 8. Compatibility of the method with different functional groups, heteroaromatic motifs, electronically and sterically varied terminal and internal alkenes, including natural products and bioactive materials, and different boronic esters. Because vinylboronic esters tend to partially decompose during chromatography on silica gel, ${ }^{20,70,71}$ the analytical yields determined by ${ }^{1} \mathrm{H}$ NMR analysis of the reaction mixture with an internal standard are reported to indicate the actual reaction performance; for the yields of isolated materials, all experimental details, and product characterization, see sections $6 \& 7$ of the SI; ${ }^{a}$ isolated yield; ${ }^{\mathrm{b}} \mathbf{4}\left(1.0\right.$ equiv) instead of $\mathbf{2}$; ${ }^{\mathrm{c}} \mathrm{B}_{2}$ pin ${ }_{2}$ as an additive $(10 \mathrm{~mol} \%) ;{ }^{\mathrm{d}}$ starting from cis-1ac, starting from trans-1ac: $38 \%$ isolated yield $(83 / 17 \mathrm{dr}) ;{ }^{\text {e }}$ The ester was readily hydrolyzed to the boronic acid (68\% yield); ${ }^{64 \mathrm{f}}$ the catalyst solution was added portion-wise (in total $8.75 \mathrm{~mol} \%$ of Rh-precursor); ${ }^{64}$ deriv, derivative.

\section{Synthetic Capacity.}

Having insight into the mechanism, we evaluated the reaction with respect to its functional group compatibility, its applicability to sterically and electronically varied olefines, including natural products and bioactive materials, as well as its ability to transfer different boryl groups (Figure 8). We established the following:

- The reaction is compatible with a broad variety of functional groups (1f-1s) and heteroaromatic moieties (1t1v). Particularly noteworthy is the compatibility with aldehydes 1f, alkynes 1r, 1s, 1w, amines 1g, 1h, and pyridines $\mathbf{1 t}, \mathbf{1} \mathbf{u}$, which are often problematic in current borylation protocols, ${ }^{6}$ due to either their ability to inhibit the catalyst or their intrinsic incompatibility with reagents (e.g., HBpin) or catalytic intermediates (e.g., $\mathrm{Ru}(\mathrm{II})$ - or $\mathrm{Rh}(\mathrm{III})$-hydrides). It is worth noting that in initial reactions of alkynes $\mathbf{1 r}$ or $\mathbf{1 s}$ with donor $\mathbf{2}$, a competitive ene-yne coupling was observed $;^{72,73}$ however, the use of vinyl $(E)$-1,2-bisboronate 4 in place of donor $\mathbf{2}$ prevented these side-reactions. Inter se, alkene $\mathbf{1} \mathbf{w}$ that contains an alkyl-benzyl alkyne moiety reacted to form boryl derivative $\mathbf{3 w}$ bearing a (non-borylated) 1,3-diene motif, revealing the alkyne-isomerization activity ${ }^{74}$ of this Rh-catalyst as well as a high selectivity of the catalyst towards terminal mono alkenes over dienes.

- The reaction is applicable to a range of olefins bearing electronically (e.g., 1x, 1y) and sterically (e.g., 1z) varied double bonds and different substitution patterns, including 1,1- and 1,2-disubstituted alkenes 1aa-1ac. In reactions of starting materials 1z-1ac, the presence of cocatalytic $\mathrm{B}_{2} \mathrm{pin}_{2}$ was essential for the efficient catalytic activity.

- The reaction is applicable to transfer different boronate ester groups, including Bmac (3ad), or the chiral $\alpha$-pinene-based Bpnd group (3ae), providing an easy access to boronic acid derivatives of varied reactivity for 
tailored synthetic applications. ${ }^{75-78}$ It is also worth noting that the method employs only simple readily available reagents and is easily scalable $(1.1 \mathrm{~g}$ of $\mathbf{3 d}$ ( $76 \%$ yield) furnished using just $0.24 \mathrm{~mol} \%$ of $\left.[\mathrm{Rh}(\operatorname{cod}) \mathrm{OMe}]_{2}\right)$.

- Typical motifs of drug molecules with multiple strongly coordinating $N$ - or $S$-sites that could chelate to the catalyst and hence impede the reaction, typically represent a significant challenge in transition metal catalysis. Nonetheless, we observed that derivatives of antihistamine Brompheniramine 1af, antipsychotic Chlorpromazine 1ag, and agonist of retinoid receptor CD3254 3ah underwent transfer borylation efficiently, establishing a powerful entry point for the synthesis of large libraries of such bioactive molecules. Additionally, boronic acids are bioisosteres of carboxylic acids that find diverse applications in pharmacology. ${ }^{79-81}$ In this context, the protocol proved effective in concise synthesis of boronic acid bioisosteres of antiplatelet drug Ozagrel 5 through its Bpin ester 3ai.

- Complex natural products of macrocyclic structure are particularly challenging and incompatible with the previous borylation methods. Gratifyingly, our protocol furnished boryl derivative 3aj of Zearalenol, a nonsteroidal mycoestrogen found in Fusarium spp. Albeit the product was formed in modest yield, this example demonstrates the unique synthetic capacity of the transfer borylation strategy.

Overall, the examples underscore the enabling potential of the approach in the late-stage functionalization of complex structures. Further studies toward increasing the catalytic capacity of the method toward other challenging alkenes are underway.

\section{CONCLUSIONS}

In conclusion, the herein disclosed $\mathrm{Rh}(\mathrm{I})$-catalyzed boryl group transfer reaction proved to be feasible and applicable not only to simple alkenes but also to more complex settings of polyfunctionalized molecules with multiple groups that could inhibit the catalyst. Therefore, such reactivity together with an excellent functional group tolerance indicates its possible applicability to late-stage modifications of complex fine chemicals. Further, the mechanistic studies provided insight into the features controlling the selectivity setting the stage for the development of methods to access different regioisomers of the products; a feature that remains elusive with current strategies.

The studies revealed that an uncommon $\beta$-boryl elimination step engaged in the reaction is notably easy, especially in contrast to the well-documented reverse step - an alkene insertion into a $\mathrm{Rh}-\mathrm{B}$ bond. Considering the number of known alkene insertion elementary reactions into different metal-heteroatom bonds, the mechanistic approach carries the potential for the development of other hydrogen-functional group exchange reactions of high value to organic synthesis. The research to uncover the full capacity of the strategy continues in our laboratories.

\section{ASSOCIATED CONTENT}

\section{Supporting Information}

Details on experimental procedures for the catalytic reactions, the mechanistic and kinetic experiments, detailed DFT data, and spectroscopic data for the starting materials and the products (PDF) Copies of NMR spectra (PDF)

DFT computed geometries in .xyz file format discussed in the text (XYZ)

\section{AUTHOR INFORMATION}

\section{Corresponding Author}

* E-mail: dydio@unistra.fr

\section{Author Contributions}

All authors have given approval to the final version of the manuscript.

†These authors contributed equally.

\section{ACKNOWLEDGMENT}

We acknowledge Amir Hoveyda (Boston College \& University of Strasbourg) and David Leboeuf (University of Strasbourg) for their comments on the manuscript, Sebastian Torker (Boston College \& University of Strasbourg) for his helpful comments on the DFT studies, Stephanie Coutin (University of Strasbourg) for the in situ HRMS analysis, and Valentin Bauer (University of Strasbourg) for the preparative HPLC purification. We acknowledge funding from the European Research Council (ERC StG no 804106), the French National Research Agency (ANR IdEx, and ANR LabEx „Chemistry of Complex Systems"), and the Frontier Research in Chemistry Foundation (an Excellence Solvay PhD fellowship for SM), and the High Performance Computing Center of the University of Strasbourg, which is partially funded by the Equipex Equip@ Meso project (Programme Investissements d'Avenir) and the CPER Alsacalcul/Big Data), for computing resources.

\section{REFERENCES}

(1) Miyaura, Norio.; Suzuki, Akira. Palladium-Catalyzed Cross-Coupling Reactions of Organoboron Compounds. Chem. Rev. 1995, 95 (7), 2457-2483. https://doi.org/10.1021/cr00039a007.

Nicolaou, K. C.; Bulger, P. G.; Sarlah, D. Palladium-Catalyzed Cross-Coupling Reactions in Total Synthesis. Angew. Chem. Int. Ed. 2005, 44 (29), 4442-4489. https://doi.org/10.1002/anie.200500368.

(3) Lennox, A. J. J.; Lloyd-Jones, G. C. Selection of Boron Reagents for Suzuki-Miyaura Coupling. Chem Soc Rev 2014, 43 (1), 412-443. https://doi.org/10.1039/C3CS60197H.

(4) Xu, L.; Zhang, S.; Li, P. Boron-Selective Reactions as Powerful Tools for Modular Synthesis of Diverse Complex Molecules. Chem. Soc. Rev. 2015, 44 (24), 8848 8858. https://doi.org/10.1039/C5CS00338E.

(5) Hartwig, J. F.; Larsen, M. A. Undirected, Homogeneous $\mathrm{C}-\mathrm{H}$ Bond Functionalization: Challenges and Opportunities. ACS Cent. Sci. 2016, 2 (5), 281-292. https://doi.org/10.1021/acscentsci.6b00032.

(6) Carreras, J.; Caballero, A.; Pérez, P. J. Alkenyl Boronates: Synthesis and Applications. Chem. - Asian J. 2019, 14 (3), 329-343. https://doi.org/10.1002/asia.201801559.

(7) Wang, X.; Wang, Y.; Huang, W.; Xia, C.; Wu, L. Direct Synthesis of Multi(Boronate) Esters from Alkenes and Alkynes via Hydroboration and Boration Reactions. ACS Catal. 2021, $11 \quad$ (1), $1-18$. https://doi.org/10.1021/acscatal.0c03418.

Flynn, A. B.; Ogilvie, W. W. Stereocontrolled Synthesis of Tetrasubstituted Olefins. Chem. Rev. 2007, 107 (11), 4698-4745. https://doi.org/10.1021/cr050051k.

(9) Blackwell, H. E.; O’Leary, D. J.; Chatterjee, A. K.; Washenfelder, R. A.; Bussmann, D. A.; Grubbs, R. H. New 
Approaches to Olefin Cross-Metathesis. J. Am. Chem. Soc. 2000, 122 (1), 58-71. https://doi.org/10.1021/ja993063u. Morrill, C.; Grubbs, R. H. Synthesis of Functionalized Vinyl Boronates via Ruthenium-Catalyzed Olefin Cross-Metathesis and Subsequent Conversion to Vinyl Halides. $J$. Org. Chem. 2003, 68 (15), 6031-6034. https://doi.org/10.1021/jo0345345.

(11) Morrill, C.; Funk, T. W.; Grubbs, R. H. Synthesis of TriSubstituted Vinyl Boronates via Ruthenium-Catalyzed Olefin Cross-Metathesis. Tetrahedron Lett. 2004, 45 (41), 7733-7736. https://doi.org/10.1016/j.tetlet.2004.08.069.

(12) Kiesewetter, E. T.; O’Brien, R. V.; Yu, E. C.; Meek, S. J.; Schrock, R. R.; Hoveyda, A. H. Synthesis of Z-(Pinacolato)Allylboron and Z-(Pinacolato)Alkenylboron Compounds through Stereoselective Catalytic Cross-Metathesis. J. Am. Chem. Soc. 2013, 135 (16), 6026-6029. https://doi.org/10.1021/ja403188t.

(13) Hemelaere, R.; Carreaux, F.; Carboni, B. Cross-Metathesis/Isomerization/Allylboration Sequence for a Diastereoselective Synthesis of Anti -Homoallylic Alcohols from Allylbenzene Derivatives and Aldehydes. Chem. - Eur. J. 2014, $20 \quad$ (44), 14518-14523. https://doi.org/10.1002/chem.201403954.

(14) Njardarson, J. T.; Biswas, K.; Danishefsky, S. J. Application of Hitherto Unexplored Macrocyclization Strategies in the Epothilone Series: Novel Epothilone Analogs by Total Synthesis. Chem. Commun. 2002, No. 23, 27592761. https://doi.org/10.1039/b209941a.

(15) Nicolaou, K. C.; Tria, G. S.; Edmonds, D. J.; Kar, M. Total Syntheses of $( \pm)$-Platencin and (-)-Platencin. J. Am. Chem. Soc. 2009, 131 (43), 15909-15917. https://doi.org/10.1021/ja906801g.

(16) Speed, A. W. H.; Mann, T. J.; O’Brien, R. V.; Schrock, R. R.; Hoveyda, A. H. Catalytic Z-Selective Cross-Metathesis in Complex Molecule Synthesis: A Convergent Stereoselective Route to Disorazole C1. J. Am. Chem. Soc. 2014, $\quad 136 \quad$ (46), 16136-16139. https://doi.org/10.1021/ja509973r.

(17) Williams, D. R.; Shah, A. A. Total Synthesis of (+)-Ileabethoxazole via an Iron-Mediated Pauson-Khand $[2+2+$ 1] Carbocyclization. J. Am. Chem. Soc. 2014, 136 (24), 8829-8836. https://doi.org/10.1021/ja5043462.

(18) Liao, L.; Zhou, J.; Xu, Z.; Ye, T. Concise Total Synthesis of Nannocystin A. Angew. Chem. Int. Ed. 2016, 55 (42), 13263-13266. https://doi.org/10.1002/anie.201606679.

(19) Boryl-Heck (ref. 20-22) and dehydrogenative borylation (ref. 23-36) protocols that are applicable to the terminal and internal alkene starting materials were also reported. However, the synthetic utility of these methods is burdened by the use of highly reactive reagents, the possibility of competitive side-reactions, and limited atom-economy. In the case of the boryl-Heck reaction (ref. 20-22), the use of highly reactive $\mathrm{Cl}-\mathrm{Bcat}$ or $\mathrm{Br}-\mathrm{Bcat}$ as the borylation reagents results in the inevitable incompatibility with many functional groups, including $\mathrm{NH}$-amines, alcohols, or enolizable ketones. Due to the low stability of the corresponding Bcat derivatives, a boronate transesterification of Bcat products to Bpin derivatives is required. In turn, the dehydrogenative borylation protocols (ref. 23-36) often suffer from competitive hydrogenation and hydroboration of the alkene starting materials diminishing their efficiency. Also, except for the unique $\mathrm{Zr}$-catalyzed protocol (ref. 36), a sacrificial hydrogen acceptor is needed to quench the metal-hydride intermediates. Therefore, starting materials containing structural motifs of higher reactivity than that of the sacrificial acceptor are inherently incompatible.
(20) Reid, W. B.; Spillane, J. J.; Krause, S. B.; Watson, D. A. Direct Synthesis of Alkenyl Boronic Esters from Unfunctionalized Alkenes: A Boryl-Heck Reaction. J. Am. Chem. Soc. 2016, 138 (17), 5539-5542. https://doi.org/10.1021/jacs.6b02914.

(21) Reid, W. B.; Watson, D. A. Synthesis of Trisubstituted Alkenyl Boronic Esters from Alkenes Using the BorylHeck Reaction. Org. Lett. 2018, 20 (21), 6832-6835. https://doi.org/10.1021/acs.orglett.8b02949.

(22) Idowu, O. O.; Hayes, J. C.; Reid, W. B.; Watson, D. A. Synthesis of 1,1-Diboryl Alkenes Using the Boryl-Heck Reaction. Org. Lett. 2021, acs.orglett.1c01567. https://doi.org/10.1021/acs.orglett.1c01567.

(23) Coapes, R. B.; Souza, F. E. S.; Thomas, R. Ll.; Hall, J. J.; Marder, T. B. Rhodium Catalysed Dehydrogenative Borylation of Vinylarenes and 1,1-Disubstituted Alkenes without Sacrificial Hydrogenation - a Route to 1,1-Disubstituted Vinylboronates. Chem. Commun. 2003, No. 5, 614-615. https://doi.org/10.1039/b211789d.

(24) Brown, J. M.; Lloyd-Jones, G. C. Vinylborane Formation in Rhodium-Catalyzed Hydroboration of Vinylarenes. Mechanism versus Borane Structure and Relationship to Silation. J. Am. Chem. Soc. 1994, 116 (3), 866-878. https://doi.org/10.1021/ja00082a006.

(25) Mkhalid, I. A. I.; Coapes, R. B.; Edes, S. N.; Coventry, D. N.; Souza, F. E. S.; Thomas, R. Ll.; Hall, J. J.; Bi, S.-W.; Lin, Z.; Marder, T. B. Rhodium Catalysed Dehydrogenative Borylation of Alkenes: Vinylboronates via $\mathrm{C}-\mathrm{H}$ Activation. Dalton Trans 2008, No. 8, 1055-1064. https://doi.org/10.1039/B715584K.

(26) Kondoh, A.; Jamison, T. F. Rhodium-Catalyzed Dehydrogenative Borylation of Cyclic Alkenes. Chem. Commun. 2010, 46 (6), 907. https://doi.org/10.1039/b921387b.

(27) Morimoto, M.; Miura, T.; Murakami, M. Rhodium-Catalyzed Dehydrogenative Borylation of Aliphatic Terminal Alkenes with Pinacolborane. Angew. Chem. Int. Ed. 2015, 54 (43), 12659-12663. https://doi.org/10.1002/anie.201506328.

(28) Selander, N.; Willy, B.; Szabó, K. J. Selective C-H Borylation of Alkenes by Palladium Pincer Complex Catalyzed Oxidative Functionalization. Angew. Chem. Int. Ed. 2010, 49 (24), 4051-4053. https://doi.org/10.1002/anie.201000690.

(29) Kirai, N.; Iguchi, S.; Ito, T.; Takaya, J.; Iwasawa, N. PSiPPincer Type Palladium-Catalyzed Dehydrogenative Borylation of Alkenes and 1,3-Dienes. Bull. Chem. Soc. $\begin{array}{llll}\text { Jpn. } & \text { 2013, } & 86 & \text { (7), }\end{array}$ https://doi.org/10.1246/bcsj.20130004.

(30) Takaya, J.; Kirai, N.; Iwasawa, N. Efficient Synthesis of Diborylalkenes from Alkenes and Diboron by a New PSiP-Pincer Palladium-Catalyzed Dehydrogenative Borylation. J. Am. Chem. Soc. 2011, 133 (33), 12980 12983. https://doi.org/10.1021/ja205186k.

(31) Sasaki, I.; Doi, H.; Hashimoto, T.; Kikuchi, T.; Ito, H.; Ishiyama, T. Iridium(i)-Catalyzed Vinylic $\mathrm{C}-\mathrm{H}$ Borylation of 1-Cycloalkenecarboxylates with Bis(Pinacolato)Diboron. Chem. Commun. 2013, 49 (68), 7546. https://doi.org/10.1039/c3cc44149k.

(32) Ohmura, T.; Takasaki, Y.; Furukawa, H.; Suginome, M. Stereoselective Synthesis of Cis- $\beta$-Methyl- and PhenylSubstituted Alkenylboronates by Platinum-Catalyzed Dehydrogenative Borylation. Angew. Chem. Int. Ed. 2009, 48 (13), 2372-2375. https://doi.org/10.1002/anie.200805406.

(33) Wang, C.; Wu, C.; Ge, S. Iron-Catalyzed $E$-Selective Dehydrogenative Borylation of Vinylarenes with Pinacolborane. ACS Catal. 2016, 6 (11), 7585-7589. https://doi.org/10.1021/acscatal.6b02654. 
Mazzacano, T. J.; Mankad, N. P. Dehydrogenative Borylation and Silylation of Styrenes Catalyzed by Copper-Carbenes. ACS Catal. 2017, 7 (1), 146-149. https://doi.org/10.1021/acscatal.6b02594.

(35) Lu, W.; Shen, Z. Direct Synthesis of Alkenylboronates from Alkenes and Pinacol Diboron via Copper Catalysis. Org. Lett. 2019, 21 (1), 142-146. https://doi.org/10.1021/acs.orglett.8b03599.

(36) Shi, X.; Li, S.; Wu, L. H 2 -Acceptorless Dehydrogenative Boration and Transfer Boration of Alkenes Enabled by Zirconium Catalyst. Angew. Chem. Int. Ed. 2019, 58 (45), 16167-16171. https://doi.org/10.1002/anie.201908931.

(37) Chatterjee, A. K.; Choi, T.-L.; Sanders, D. P.; Grubbs, R. H. A General Model for Selectivity in Olefin Cross Metathesis. J. Am. Chem. Soc. 2003, 125 (37), 11360-11370. https://doi.org/10.1021/ja0214882.

(38) Nascimento, D. L.; Reim, I.; Foscato, M.; Jensen, V. R.; Fogg, D. E. Challenging Metathesis Catalysts with Nucleophiles and Brønsted Base: Examining the Stability of State-of-the-Art Ruthenium Carbene Catalysts to Attack by Amines. ACS Catal. 2020, 10 (19), 11623-11633. https://doi.org/10.1021/acscatal.0c02760.

(39) Bailey, G. A.; Lummiss, J. A. M.; Foscato, M.; Occhipinti, G.; McDonald, R.; Jensen, V. R.; Fogg, D. E. Decomposition of Olefin Metathesis Catalysts by Brønsted Base: Metallacyclobutane Deprotonation as a Primary Deactivating Event. J. Am. Chem. Soc. 2017, 139 (46), 1644616449. https://doi.org/10.1021/jacs.7b08578.

(40) Lafaye, K.; Nicolas, L.; Guérinot, A.; Reymond, S.; Cossy, J. Lewis Basicity Modulation of $N$-Heterocycles: A Key for Successful Cross-Metathesis. Org. Lett. 2014, 16 (19), 4972-4975. https://doi.org/10.1021/ol502016h.

(41) P'Poo, S. J.; Schanz, H.-J. Reversible Inhibition/Activation of Olefin Metathesis: A Kinetic Investigation of ROMP and RCM Reactions with Grubbs' Catalyst. J. Am. Chem. Soc. 2007, 129 (46), 14200-14212. https://doi.org/10.1021/ja071938w.

(42) Mann, T. J.; Speed, A. W. H.; Schrock, R. R.; Hoveyda, A. H. Catalytic $Z$-Selective Cross-Metathesis with Secondary Silyl- and Benzyl-Protected Allylic Ethers: Mechanistic Aspects and Applications to Natural Product Synthesis. Angew. Chem. Int. Ed. 2013, 52 (32), 8395-8400. https://doi.org/10.1002/anie.201302538.

(43) Xu, Y.; Wong, J. J.; Samkian, A. E.; Ko, J. H.; Chen, S.; Houk, K. N.; Grubbs, R. H. Efficient $Z$-Selective OlefinAcrylamide Cross-Metathesis Enabled by Sterically Demanding Cyclometalated Ruthenium Catalysts. J. Am. Chem. Soc. 2020, 142 (50), 20987-20993. https://doi.org/10.1021/jacs.0c11334.

(44) Koh, M. J.; Khan, R. K. M.; Torker, S.; Yu, M.; Mikus, M. S.; Hoveyda, A. H. High-Value Alcohols and Higher-Oxidation-State Compounds by Catalytic Z-Selective CrossMetathesis. Nature 2015, 517 (7533), 181-186. https://doi.org/10.1038/nature14061.

(45) Bhawal, B. N.; Morandi, B. Catalytic Isofunctional Reactions-Expanding the Repertoire of Shuttle and Metathesis Reactions. Angew. Chem. Int. Ed. 2019, 58 (30), 10074-10103. https://doi.org/10.1002/anie.201803797.

(46) Rochette, É.; Desrosiers, V.; Soltani, Y.; Fontaine, F.-G. Isodesmic $\mathrm{C}-\mathrm{H}$ Borylation: Perspectives and Proof of Concept of Transfer Borylation Catalysis. J. Am. Chem. $\begin{array}{lllll}\text { Soc. } & \mathbf{2 0 1 9}, & 141 & \text { (31), } & 12305-12311 .\end{array}$ https://doi.org/10.1021/jacs.9b04305.

(47) Marciniec, B.; Jankowska, M.; Pietraszuk, C. New Catalytic Route to Functionalized Vinylboronates. Chem. Commun. 2005, No. 5, 663. https://doi.org/10.1039/b414644a.
(48) Marciniec, B.; Dudziec, B.; Kownacki, I. A New Catalytic Route for the Activation of Sp-Hybridized Carbon-Hydrogen Bonds. Angew. Chem. Int. Ed. 2006, 45 (48), 8180-8184. https://doi.org/10.1002/anie.200603582.

(49) Lam, K. C.; Lin, Z.; Marder, T. B. DFT Studies of $\beta$-Boryl Elimination Processes: Potential Role in Catalyzed Borylation Reactions of Alkenes. Organometallics 2007, 26 (13), 3149-3156. https://doi.org/10.1021/om0700314.

(50) Kalläne, S. I.; Braun, T.; Braun, B.; Mebs, S. Versatile Reactivity of a Rhodium(i) Boryl Complex towards Ketones and Imines. Dalton Trans. 2014, 43 (18), 6786. https://doi.org/10.1039/c4dt00080c.

(51) Franke, R.; Selent, D.; Börner, A. Applied Hydroformylation. Chem. Rev. 2012, 112 (11), 5675-5732. https://doi.org/10.1021/cr3001803.

(52) Murphy, S. K.; Park, J.-W.; Cruz, F. A.; Dong, V. M. RhCatalyzed $\mathrm{C}-\mathrm{C}$ Bond Cleavage by Transfer Hydroformylation. Science 2015, 347 (6217), 56-60. https://doi.org/10.1126/science.1261232.

(53) Wu, X.; Cruz, F. A.; Lu, A.; Dong, V. M. Tandem Catalysis: Transforming Alcohols to Alkenes by Oxidative Dehydroxymethylation. J. Am. Chem. Soc. 2018, 140 (32), 10126-10130. https://doi.org/10.1021/jacs.8b06069.

(54) Tan, G.; Wu, Y.; Shi, Y.; You, J. Syngas-Free Highly Regioselective Rhodium-Catalyzed Transfer Hydroformylation of Alkynes to $\alpha, \beta$-Unsaturated Aldehydes. Angew. Chem. Int. Ed. 2019, 58 (22), 7440-7444. https://doi.org/10.1002/anie.201902553.

(55) Baker, R. T.; Calabrese, J. C.; Westcott, S. A.; Nguyen, P.; Marder, T. B. Insertion of Alkenes into Rhodium-Boron Bonds. J. Am. Chem. Soc. 1993, 115 (10), 4367-4368. https://doi.org/10.1021/ja00063a067.

(56) Teltewskoi, M.; Panetier, J. A.; Macgregor, S. A.; Braun, T. A Highly Reactive Rhodium(I)-Boryl Complex as a Useful Tool for C-H Bond Activation and Catalytic C-F Bond Borylation. Angew. Chem. Int. Ed. 2010, 49 (23), 3947-3951. https://doi.org/10.1002/anie.201001070.

(57) Dai, C.; Stringer, G.; Marder, T. B.; Scott, A. J.; Clegg, W.; Norman, N. C. Synthesis and Characterization of Rhodium(I) Boryl and Rhodium(III) Tris(Boryl) Compounds: Molecular Structures of $\left[\left(\mathrm{PMe}_{3}\right)_{4} \mathrm{Rh}(\mathrm{B}(\mathrm{Cat}))\right]$ and $\mathrm{Fac}-$ $\left[\left(\mathrm{PMe}_{3}\right)_{3} \mathrm{Rh}(\mathrm{B}(\mathrm{Cat}))_{3}\right]\left(\mathrm{Cat}=1,2-\mathrm{O}_{2} \mathrm{C}_{6} \mathrm{H}_{4}\right)$. Inorg. Chem. 1997, $36 \quad$ (3), 272-273. https://doi.org/10.1021/ic9611047.

(58) Masuda, Y.; Hasegawa, M.; Yamashita, M.; Nozaki, K.; Ishida, N.; Murakami, M. Oxidative Addition of a Strained C-C Bond onto Electron-Rich Rhodium(I) at Room Temperature. J. Am. Chem. Soc. 2013, 135 (19), 7142-7145. https://doi.org/10.1021/ja403461f.

(59) Irvine, G. J.; Lesley, M. J. G.; Marder, T. B.; Norman, N. C.; Rice, C. R.; Robins, E. G.; Roper, W. R.; Whittell, G. R.; Wright, L. J. Transition Metal-Boryl Compounds: Synthesis, Reactivity, and Structure. Chem. Rev. 1998, 98 (8), 2685-2722. https://doi.org/10.1021/cr9500085.

(60) Braunschweig, H.; Colling, M. Transition Metal Complexes of Boron - Synthesis, Structure and Reactivity. Coord. Chem. Rev. 2001, 223 (1), 1-51. https://doi.org/10.1016/S0010-8545(01)00378-2.

(61) Esteruelas, M. A.; Oliván, M.; Vélez, A. POP-RhodiumPromoted $\mathrm{C}-\mathrm{H}$ and $\mathrm{B}-\mathrm{H}$ Bond Activation and $\mathrm{C}-\mathrm{B}$ Bond Formation. Organometallics 2015, 34 (10), 1911-1924. https://doi.org/10.1021/acs.organomet.5b00176.

(62) Zhao, P.; Incarvito, C. D.; Hartwig, J. F. Carbon-Oxygen Bond Formation between a Terminal Alkoxo Ligand and a Coordinated Olefin. Evidence for Olefin Insertion into a Rhodium Alkoxide. J. Am. Chem. Soc. 2006, 128 (30), 9642-9643. https://doi.org/10.1021/ja063347w. 
(63) Richers, C. P.; Roediger, S.; Laserna, V.; Hartwig, J. F. Effects of Ligands on the Migratory Insertion of Alkenes into Rhodium-Oxygen Bonds. Chem. Sci. 2020, 11 (38), 10449-10456. https://doi.org/10.1039/D0SC04402D.

(64) For details, see the Supporting Information.

(65) Kloek, S. M.; Heinekey, D. M.; Goldberg, K. I. C-H Bond Activation by Rhodium(I) Hydroxide and Phenoxide Complexes. Angew. Chem. Int. Ed. 2007, 46 (25), 47364738. https://doi.org/10.1002/anie.200700270.

(66) Kegley, S. E.; Schaverien, C. J.; Freudenberger, J. H.; Bergman, R. G.; Nolan, S. P.; Hoff, C. D. Rhodium Alkoxide Complexes. Formation of an Unusually Strong Intermolecular Hydrogen Bond in (PMe3)3RhOtol(HOtol). J. Am. Chem. Soc. 1987, 109 (21), 6563 6565. https://doi.org/10.1021/ja00255a080.

(67) Qi, X.; Li, Y.; Bai, R.; Lan, Y. Mechanism of RhodiumCatalyzed C-H Functionalization: Advances in Theoretical Investigation. Acc. Chem. Res. 2017, 50 (11), 27992808. https://doi.org/10.1021/acs.accounts.7b00400.

(68) Uetake, Y.; Niwa, T.; Hosoya, T. Rhodium-Catalyzed Ipso -Borylation of Alkylthioarenes via $\mathrm{C}-\mathrm{S}$ Bond Cleavage. Org. Lett. 2016, 18 (11), 2758-2761. https://doi.org/10.1021/acs.orglett.6b01250.

(69) Bhawal, B. N.; Reisenbauer, J. C.; Ehinger, C.; Morandi, B. Overcoming Selectivity Issues in Reversible Catalysis: A Transfer Hydrocyanation Exhibiting High Kinetic Control. J. Am. Chem. Soc. 2020, 142 (25), 10914-10920. https://doi.org/10.1021/jacs.0c03184.

(70) Du, R.; Liu, L.; Xu, S. Iridium-Catalyzed Regio- and Enantioselective Borylation of Unbiased Methylene C(Sp ${ }^{3}$ ) $-\mathrm{H}$ Bonds at the Position $\beta$ to a Nitrogen Center. Angew. Chem. Int. Ed. 2021, 60 (11), 5843-5847. https://doi.org/10.1002/anie.202016009.

(71) Hitosugi, S.; Tanimoto, D.; Nakanishi, W.; Isobe, H. A Facile Chromatographic Method for Purification of Pinacol Boronic Esters. Chem. Lett. 2012, 41 (9), 972-973. https://doi.org/10.1246/cl.2012.972.

(72) Lindhardt (neé Hansen), A. T.; Mantel, M. L. H.; Skrydstrup, T. Palladium-Catalyzed Intermolecular EneYne Coupling: Development of an Atom-Efficient
Mizoroki-Heck-Type Reaction. Angew. Chem. Int. Ed. 2008, $\quad 47 \quad$ (14), 2668-2672. https://doi.org/10.1002/anie.200705558.

(73) Kaminsky, L.; Wilson, R. J.; Clark, D. A. Stereo- and Regioselective Formation of Silyl-Dienyl Boronates. Org. Lett. $\quad 2015, \quad 17 \quad$ (12), 3126-3129. https://doi.org/10.1021/acs.orglett.5b01434.

(74) Shintani, R.; Duan, W.-L.; Park, S.; Hayashi, T. RhodiumCatalyzed Isomerization of Unactivated Alkynes to 1,3Dienes. Chem. Commun. 2006, No. 34, 3646. https://doi.org/10.1039/b605368h.

(75) Myhill, J. A.; Wilhelmsen, C. A.; Zhang, L.; Morken, J. P. Diastereoselective and Enantioselective Conjunctive Cross-Coupling Enabled by Boron Ligand Design. J. Am. Chem. Soc. 2018, 140 (45), 15181-15185. https://doi.org/10.1021/jacs.8b09909.

(76) Hecker, S. J.; Reddy, K. R.; Lomovskaya, O.; Griffith, D. C.; Rubio-Aparicio, D.; Nelson, K.; Tsivkovski, R.; Sun, D.; Sabet, M.; Tarazi, Z.; Parkinson, J.; Totrov, M.; Boyer, S. H.; Glinka, T. W.; Pemberton, O. A.; Chen, Y.; Dudley, M. N. Discovery of Cyclic Boronic Acid QPX7728, an Ultrabroad-Spectrum Inhibitor of Serine and Metallo- $\beta$-Lactamases. J. Med. Chem. 2020, 63 (14), 7491-7507. https://doi.org/10.1021/acs.jmedchem.9b01976.

(77) Matteson, D. S. Boronic Esters in Asymmetric Synthesis. J. Org. Chem. 2013, 78 (20), 10009-10023. https://doi.org/10.1021/jo4013942.

(78) Andrés, P.; Ballano, G.; Calaza, M. I.; Cativiela, C. Synthesis of $\alpha$-Aminoboronic Acids. Chem. Soc. Rev. 2016, 45 (8), 2291-2307. https://doi.org/10.1039/C5CS00886G.

(79) Hall, D. G. Boronic Acids: Preparation and Applications in Organic Synthesis and Medicine; Wiley-VCH Verlag: Weinheim, 2010.

(80) Ban, H. S.; Nakamura, H. Boron-Based Drug Design: Boron-Based Drug Design. Chem. Rec. 2015, 15 (3), 616635. https://doi.org/10.1002/tcr.201402100.

(81) Diaz, D. B.; Yudin, A. K. The Versatility of Boron in Biological Target Engagement. Nat. Chem. 2017, 9 (8), 731742. https://doi.org/10.1038/nchem.2814.

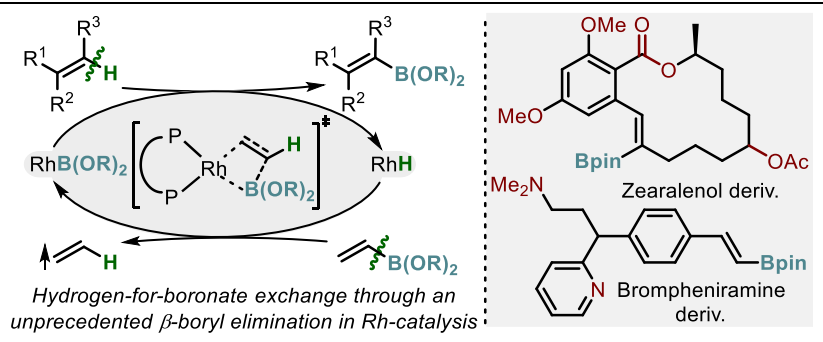

Table of Contents artwork 